\title{
A Well-Mixed, Polymer-Based Microbioreactor With Integrated Optical Measurements
}

\author{
Zhiyu Zhang, ${ }^{1}$ Nicolas Szita, ${ }^{1}$ Paolo Boccazzi, ${ }^{2}$ Anthony J. Sinskey, ${ }^{2}$ Klavs F. Jensen ${ }^{1}$ \\ ${ }^{1}$ Department of Chemical Engineering, Massachusetts Institute of Technology, \\ 77 Mass. Ave., 66-566, Cambridge, Massachusetts 02139; telephone: +1(617)253-4589; \\ fax:+1(617)258-8224; e-mail: kfjensen@mit.edu \\ ${ }^{2}$ Department of Biology and Health Sciences and Technology, Massachusetts Institute \\ of Technology, 77 Mass. Ave., 68-370A, Cambridge, Massachusetts 02139
}

Received 4 May 2005; accepted 1 August 2005

Published online 6 December 2005 in Wiley InterScience (www.interscience.wiley.com). DOI: 10.1002/bit.20678

\begin{abstract}
We describe a $150 \mu \mathrm{L}$ microbioreactor fabricated in poly(methylmethacrylate) (PMMA) and poly(dimethylsiloxane) (PDMS) to cultivate microbial cell cultures. Mixing is achieved by a small magnetic stir bar and fluorescent sensors are integrated for on-line measurement of $\mathrm{pH}$ and dissolved oxygen. Optical transmission measurements are used for cell density. The body of the reactor is poly(methylmethacrylate) with a thin layer of poly (dimethylsiloxane) for aeration, oxygen diffuses through this gas-permeable membrane into the microbioreactor to support metabolism of bacterial cells. Mixing in the reactor is characterized by observation of mixing of dyes and computational fluid dynamics simulations. The oxygenation is described in terms of measured $K_{\mathrm{L}}$ a values for microbioreactor, $20-75 / \mathrm{h}$ corresponding to increasing stirring speed $200-800 \mathrm{rpm}$. Escherichia coli cell growth in the microbioreactor is demonstrated and the growth behavior is benchmarked with conventional bench-scale bioreactors, flasks and tubes. Batch culture experiments with Saccharomyces cerevisiae further demonstrate the reproducibility and flexibility of the microbioreactor system. (c) 2005 Wiley Periodicals, Inc.

Keywords: microbioreactor; batch fermentation; $K_{\mathrm{L}} a$ measurement; membrane aeration
\end{abstract}

\section{INTRODUCTION}

There is great interest in the development of new microbial cell cultivation technologies for high-throughput screening as well as for bioprocess development. Instrumented stirredtank bioreactors effectively control temperature, $\mathrm{pH}$, and dissolved oxygen (DO) levels by controlling mixing and gas sparging rate, thus yielding valuable physiological and metabolic information at different stages of the fermentation. However, fermentation experiments using conventional

Correspondence to: Klavs F. Jensen

Nicolas Szita's present address is MIC, Department of Micro and Nanotechnology, Technical University of Denmark (DTU), DK-2800 Kgs. Lyngby, Denmark.

Contract grant sponsors: DuPont-MIT Alliance (DMA); Swiss National Science Foundation stirred-tank bioreactors are typically expensive and laborintensive for screening purposes. Improvements have been made in lab-scale stirred-tank bioreactors including reducing the reactor volume and increasing the number of reactors operating in parallel (Weuster-Botz et al., 2002), but many of these solutions require efforts, such as sterilization, assembly, cleaning, and calibration of sensors in reactors, which scale with the number of bioreactors. Thus, the need remains for developing bioreactor systems that will enable parallel experimentation with only minimum increase in handling and operational efforts.

Test tubes, shake flasks, and microtiter plates are widely used as simple bioreactors for fermentation and cell culture experiments. These bioreactors can easily be operated in parallel and with small volumes, but they offer little control over bioprocess conditions and the data obtained are often limited to endpoint measurements. Efforts have been made to implement on-line $\mathrm{pH}$ or DO measurements units in microtiter plates (John et al., 2003a,b; Weiss et al., 2002) or shake flasks (Gupta and Rao, 2003; Tolosa et al., 2002; WeusterBotz et al., 2001; Wittmann et al., 2003) to obtain continuous process information during fermentation.

Microbioreactors with integrated sensors combine the small volumes of microtiter plates with the monitoring and control features found in bench-scale systems to form promising tools for rapid, high-throughput, and cost-effective screening. Kumar et al. (2004) have reviewed recent progress in the area of minibioreactors as well as small-scale conventional bioreactors.

One fundamental requirement for milliliter- or microliterscale bioreactors is the ability to obtain optical density (OD), $\mathrm{pH}$, and $\mathrm{DO}$ data in real time and thereby avoid the need for sample removal. Recent developments in electrochemical and optical sensor technologies have made such measurements possible in microbioreactors. Walther et al. (1994, 1999, 2000) realized a $3 \mathrm{~mL}$ bioreactor with integrated biomass, $\mathrm{pH}$, and temperature microelectronic sensors for growing yeast cell cultures in space. In this bioreactor, 
$\mathrm{pH}$ was measured by a miniaturized ion-selective field effect transistor (ISFET) sensor and controlled by coulometric generation of hydroxyl ions at a titanium electrode in the bioreactor. Kim and Lee (1998) proposed a microfermentor chip that uses microelectrodes to measure biomass, $\mathrm{pH}, \mathrm{DO}$, and glucose concentration. Van der Weide and Blattner (2002) correlated capacitance and resistance to the biomass in microbial growth assay wells with a liquid content of up to $30 \mu \mathrm{L}$. The microbioreactor developed by Maharbiz et al. (2003) combined plastic microplate reactors with silicon microfabrication technology. Electrochemical sensors and capacitance measurements were constructed in the silicon and electodes of the silicon generated by hydrolysis of water. Most recently, Maharbiz et al. (2004) have integrated $250 \mu \mathrm{L}$ microbioreactor arrays with OD measurement and pH ISFET sensors on a commercial printed circuit board.

Rao and co-authors (Harms et al., 2002; Kermis et al., 2003; Kostov et al., 2001; Rao, 2002) pioneered optical measurements of $\mathrm{OD}, \mathrm{pH}$, and $\mathrm{DO}$, which are non-invasive, easily integrated, and reliable-advantageous features for high-throughput screening. In the minibioreactor by Kostov et al. (2001), fermentation parameters were measured on-line continuously; cell growth was compared with a bench-scale $1 \mathrm{~L}$ fermentor. Optical sensors were also used in the $6 \mathrm{~mL}$ miniature bioreactor reported by Lamping et al. (2003), as well as in the microbioreactor described by Zanzotto et al. (2004), in which stable signals were obtained in a $5 \mu \mathrm{L}$ microbioreactor. Commercialized applications of the optical sensors includes the Cellstation ${ }^{\circledR}$ bioreactors developed by Fluorometrix Corp. (Stow, MA), in which 12 miniature stirred-tank bioreactors are operated in parallel. Most recently, Puskeiler et al. (2005) reported parallel operation of 48 magnetically-mixed milliliter scale bioreactors arrays. In these bioreactors, $\mathrm{OD}$ and $\mathrm{pH}$ were monitored in line; automation of fed-batch operation and $\mathrm{pH}$ control were implemented by using commercially available liquid-handling systems. These efforts in miniaturization and parallelization of microbioreactors, as well as automation of measurements and controls, demonstrate the potential for obtaining valuable growth kinetics data from microbioreactors and indicate the promise of high-throughput technologies for bioprocessing.

Oxygenation is an important aspect in bioreactor design and performance. In most stirred-tank bioreactors, air or oxygen gas bubbles are sparged into the culture medium to provide microbial cells with the oxygen necessary for growth or maintenance of cellular functions. For example, the miniature bioreactor by Lamping et al. (2003) machined in Plexiglas and outfitted with air spargers and stirring baffles, can be seen as a scaled-down version of conventional benchscale stirred-tank bioreactors. Sparging of air/oxygen, such as the oxygen bubbles created in the microbioreactor by Maharbiz et al. (2004), also contributes to mixing. Shaking is another way to improve gas-liquid oxygen transportation in bioreactors (Duetz et al., 2000, 2001; Wittmann et al., 2003). Zanzotto et al. (2004) provided oxygenation to cells growing in the microbioreactor by diffusion through a thin, gas- permeable poly(dimethylsiloxane) (PDMS) membrane. The PDMS membrane separates gas and liquid phases thereby eliminating the risk of contamination from air and cross-talk between adjacent microbioreactors. Diffusion in the PDMS is sufficiently fast that the mass transfer is not limited by the PDMS membrane, but by diffusion into the broth so that an oxygen concentration gradient develops through the depth of the reactor (Zanzotto et al., 2004). As a result, such reactors have shallow depth (e.g., a few hundred microns) and the volume can only be increased by enlarging the diameter, which creates potential difficulties in membrane design.

In this study, we present a well-mixed microbioreactor as an extension of the membrane-aerated microbioreactor. Active mixing is achieved by using a mini magnetic stir bar. A volume of $150 \mu \mathrm{L}$ in the microbioreactor makes sampling possible for bioanalysis, such as HPLC or global gene expression analysis (Boccazzi et al., 2005). Construction of the microbioreactor in layers of poly(methylmethacrylate) (PMMA) and PDMS makes the reactor transparent for optical measurements and rigid for handling while preserving the aeration and aseptic properties of the PDMS membrane. Moreover, the microfabrication is compatible with large-scale plastic fabrication processes such as injection-molding and hot-embossing. Optical sensors for on-line $\mathrm{OD}, \mathrm{pH}$, and $\mathrm{DO}$ measurements are integrated into the microbioreactor. Evaporation of water during operation, a critical issue, particular for small reactors and for slowgrowing microorganisms (Kumar et al., 2004), is compensated by connecting the microbioreactor to a pressurized water reservoir maintaining the reactor volume constant during experiments. Growth of Escherichia coli and Saccharomyces cerevisiae are used as model systems. The oxygen and growth performance of the microbioreactor is characterized and benchmarked against conventional benchscale cell culture systems (e.g., test tubes, shaking flasks, and $500 \mathrm{~mL}$ stirred-tank bioreactors).

\section{EXPERIMENTAL METHODS}

\section{Microbioreactor Design}

The microbioreactor body consisted of two PMMA layers with two PDMS layers for aeration and sealing sandwiched in between them (see Fig. 1). The bottom PMMA layer, made by using a computer-numerical-controlled (CNC) milling machine, included the microbioreactor chamber (diameter $10 \mathrm{~mm}$, depth $1 \mathrm{~mm}$ ) and three connecting channels (depth $500 \mu \mathrm{m}$, width $500 \mu \mathrm{m}$ ), used for inoculation and replenishment of water. A thin layer $(100 \mu \mathrm{m})$ of spin-coated PDMS (Sylgard 184, Dow Corning Corp., Midland, MI; mixing ratio of silicone to curing agent $10: 1$; baked at $70^{\circ} \mathrm{C}$ for $2 \mathrm{~h}$ ) covered the chamber and served as the aeration membrane. The thin PDMS layer was held by a $5 \mathrm{~mm}$ thick PDMS gasket layer to facilitate device assembly, hermetical sealing, and connection of microfluidic channels.

Two recesses (diameter $2 \mathrm{~mm}$, depth $250 \mu \mathrm{m}, 2.7 \mathrm{~mm}$ radial distance from the center) at the bottom of the 

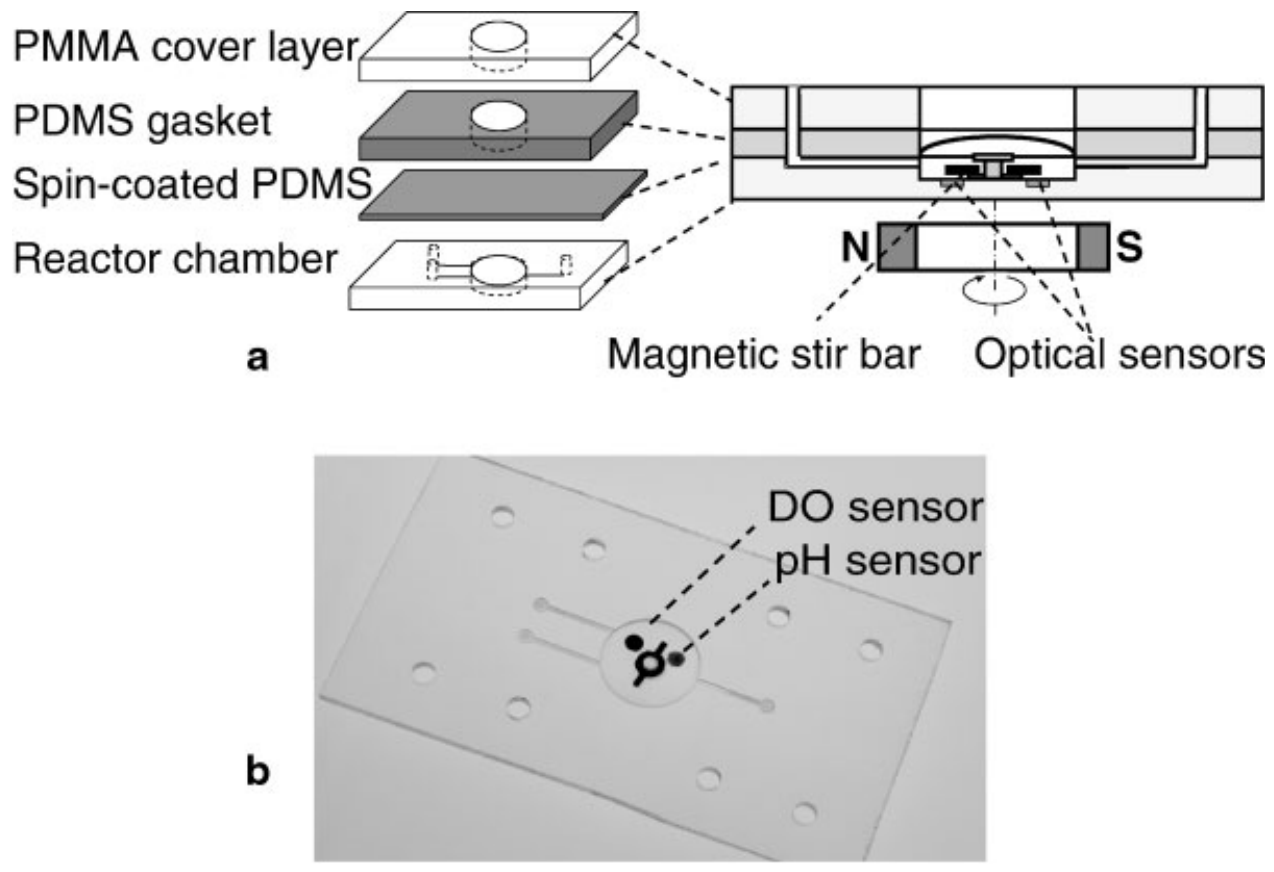

Figure 1. a: Schematic view of the lengthwise cross-section of the microbioreactor; (b) photograph of the empty poly(methylmethacrylate) (PMMA) chamber of the reactor with the magnetic stir bar in the center and dissolved oxygen (DO) and pH fluorescent sensors at the bottom of the chamber.

bioreactor chamber accommodated $\mathrm{pH}$ and $\mathrm{DO}$ fluorescence lifetime sensors (DO sensor foil PSt 3 , and $\mathrm{pH}$ sensor solution HP2A, PreSens-Precision Sensing GmbH, Regensburg, Germany). For mixing, a ring-shape magnetic stir bar with an arm length of $6 \mathrm{~mm}$ and a thickness of $0.5 \mathrm{~mm}$ (custom-made by Engineered Concepts, Vestavia Hills, AL) rotated in the center of the reactor chamber. The rotation axis was defined by a vertical post (height of $800 \mu \mathrm{m}$, diameter of $1.35 \mathrm{~mm}$ ) machined out of the bulk PMMA in the center of reactor chamber. A piece of PMMA with $250 \mu \mathrm{m}$ thick and $3 \mathrm{~mm}$ in diameter was attached on top of the PMMA post by using acrylic solvent (Weld-On 4, IPS Corp., Gardena, CA) to keep the magnetic stir bar in position (Fig. 1). By residing the stir bar on a shallow shoulder (height of $200 \mu \mathrm{m}$, diameter of $2.2 \mathrm{~mm}$ ) machined out of bulk PMMA at the bottom of the reactor, the friction between the stir bar and PMMA surface was minimized.

Polyethylene tubing (1/32" outer diameter, Becton Dickinson and Company, Franklin Lakes, NJ) were inserted into small holes punched through the $5 \mathrm{~mm}$ PDMS layer by using fluidic needle adapters (20 gauge, Becton Dickinson and Company) to connect to the channels (two inlets, one outlet) in the bottom of PMMA device. In fermentation experiments, syringes were used to inoculate the microbioreactor through one of the inlet channels. The other inlet channel was connected to an elevated external water reservoir. At $300 \mathrm{~mm}$ water pressure, the thin PDMS membrane bulged slightly upward, yielding a total volume of approximately $150 \mu \mathrm{L}$. Water was passively replenished into the microbioreactor at the same rate as water evaporated through the highly permeable PDMS thin membrane, thus keeping the volume of the microbioreactor constant during fermentation. By measuring the weight increase of anhy- drous calcium sulfate pellets (W.A. Hammond Drierite, Xenia, $\mathrm{OH}$ ) placed in a closed chamber with the microbioreactor, the water evaporation rate from the microbioreactor was determined to be $4.3 \pm 0.4 \mu \mathrm{L} / \mathrm{h}$ at $37^{\circ} \mathrm{C}$. Thus, there would be a significant loss in fluids in the bioreactor if water had not been replenished. The use of passive feeding of water makes it possible to run the $150 \mu \mathrm{L}$ bioreactor for long periods of time without an observable volume change.

The device shown in Figure 1 was used for E. coli fermentations. For S. cerevisiae cell culture experiments, in which oxygen requirement is less demanding due to longer generation time, we used a bioreactor covered with an additional layer of stainless steel grid (B-PMX-062, Small Parts, Inc., Miami, FL) to provide a rigid top perforated membrane structure.

\section{Optical Measurement Setup}

The experimental set-up is shown in Figure 2. Dissolved oxygen (DO), $\mathrm{pH}$, and optical density (OD) were measured by the optical sensing methods described in detail in Zanzotto et al. (2004), so only a brief summary is included here. Fermentations were carried out by placing the microbioreactor in an aluminum chamber maintained at $37^{\circ} \mathrm{C}$ by flowing heated water through the chamber base. An external magnetic stirrer (Thermolyne Mirak, SP72725, Barnstead International, Dubuque, IA; placed directly below the aluminum chamber.) controlled the stir bar in the microbioreactor. Bifurcated optical fibers (custom-made by RoMack Fiber Optics, Williamsburg, VA) led into the chamber from both the top and the bottom and connected to LEDs and photodetectors (PDA-55, Thorlabs, Newton, $\mathrm{NJ})$ to perform the optical measurements. Biomass was 


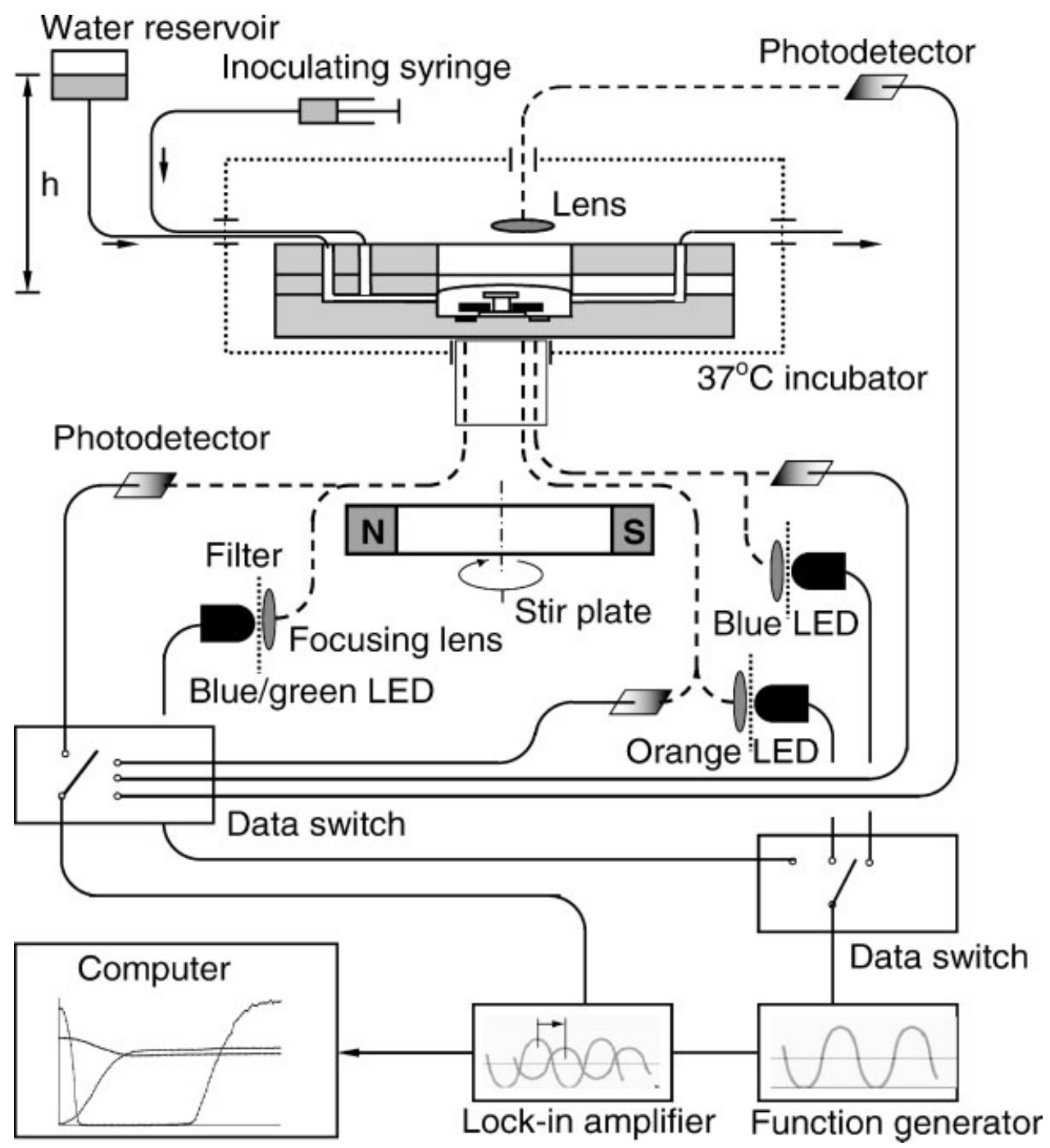

Figure 2. Experimental setup for the microbioreactor. The microbioreactor is kept at $37^{\circ} \mathrm{C}$ in an aluminum chamber; three optical fibers carry different wavelengths of light to the bottom of the microbioreactor for optical density (OD), DO, and pH measurements, respectively. A computer collects and analyzes the transmitted or emitted light through photodetectors and a lock-in amplifier. (The water reservoir is at a height, $\mathrm{h}=0.3 \mathrm{~m}$ ).

followed by OD data obtained from a transmission measurement using an orange LED (Epitex L600-10V, $600 \mathrm{~nm}$, Kyoto, Japan). The bifurcated branch provided a reference signal to compensate for any intensity fluctuations of the orange LED. Both dissolved oxygen and $\mathrm{pH}$ were measured using phase modulation lifetime fluorimetry. The $\mathrm{DO}$ and $\mathrm{pH}$ sensors were excited with a blue-green LED (505 nm, NSPE590S, Nichia America Corporation, Mountville, PA) and a blue LED (465 nm, NSPB500S, Nichia), respectively. Excitation bandpass filters (XF1016 and XF1014, Omega Optical, Inc., Brattleboro, VT) and emission long pass filters (XF 3016 and XF 3018, Omega Optical) separated the respective excitation and emission signals to minimize crossexcitation. Data switches (8037, Electro Standard Laboratories, Cranston, RI) multiplexed the output signal and the input signal of the function generator (33220A, Agilent Technologies, Palo Alto, CA) and the lock-in amplifier (SR 830, Stanford Research Systems, Sunnyvale, CA). All instruments were computer controlled under LabVIEW ${ }^{\circledR}$
(National Instruments Corp., Austin, TX), which enabled automated and real-time measurement of the parameters.

\section{Material and Methods for Biological Experiments}

E. coli FB21591 (thiC::Tn5-pKD46, Kan ${ }^{\mathrm{R}}$ ) obtained from University of Wisconsin was used as a model organism. $E$. coli cells were cultured in Luria-Bertani (LB) medium with $8 \mathrm{~g} / \mathrm{L}$ glucose (Mallinckrodt, Hazelwood, MO), $100 \mu \mathrm{g} / \mathrm{L}$ kanamycin (Sigma-Aldrich, Co., St. Louis, MO), and $0.1 \mathrm{~mol} / \mathrm{L}$ 2-( $N$-morpholino)ethanesulfonic acid (MES) (Sigma-Aldrich). Exposure to $\gamma$-radiation was proven by experiments to be effective for sterilization for the building materials of our microbioreactor without noticeable changes in properties. However, in order to simplify the bench-marking experiments, kanamycin was added to the culture medium to prevent contamination from other bacteria species.

To ensure reproducible inoculation for different fermentation experiments, the cell cultures were prepared according 
to a standardized protocol: single colonies of $E$. coli FB21591 were transferred from LB plates (containing $2 \%$ (wt/vol) agar and $100 \mu \mathrm{g} / \mathrm{L}$ of kanamycin) to $5 \mathrm{~mL}$ of sterile $\mathrm{LB}$ medium (containing $8 \mathrm{~g} / \mathrm{L}$ glucose, $100 \mu \mathrm{g} / \mathrm{L}$ kanamycin, and $0.1 \mathrm{~mol} /$ L MES) in test tubes. These cultures were then incubated on a roller drum at $60 \mathrm{rpm}$ and $37^{\circ} \mathrm{C}$. When the culture reached an $\mathrm{OD}_{600 \mathrm{~nm}}$ of $1,1.5 \mathrm{~mL}$ of culture medium was transferred from test tubes to $30 \mathrm{~mL}$ of $\mathrm{LB}$ medium in a $250 \mathrm{~mL}$ baffled shake flask. The shake flask was incubated on a horizontal rotary shaker (150-220 rpm, Lab-line 4690, Barnstead International) at $37^{\circ} \mathrm{C}$ until an $\mathrm{OD}_{600 \mathrm{~nm}}$ of 1 was again reached. The culture medium in the shake flask was diluted in fresh medium to match an $\mathrm{OD}_{600 \mathrm{~nm}}$ of 0.05 and used to inoculate the different bioreactors. Except for the microbioreactor, optical density measurements were performed off-line in a spectrophotometer (Spectronic 20 Genesys, Spectronic Instruments, Leeds, UK). We used conventional cultivation techniques, including test tubes, baffled shake flasks, and 500-mL stirred-tank (Sixfors ${ }^{\circledR}$, Infors AG) to benchmark E. coli batch cultures in the microbioreactor.

For bench-marking experiments with test tubes, a total of 13 tubes were filled with $5 \mathrm{~mL}$ of the same inoculum derived from a single colony. The tubes were incubated on a roller drum at $60 \mathrm{rpm}$ and $37^{\circ} \mathrm{C}$. Single test tubes were sacrificed at 13 different time points over an $18 \mathrm{~h}$ time period to measure $\mathrm{pH}$ and OD. The experiment was replicated with four independent inoculations. For bench-marking experiments in shake flasks $(1 \mathrm{~L}), \mathrm{pH}$ and OD readings were obtained by taking $1 \mathrm{~mL}$ samples at different time points from shake flasks filled with $150 \mathrm{~mL}$ of inoculum. Shake flasks were incubated at $37^{\circ} \mathrm{C}$ with a shaking speed of $200 \mathrm{rpm}$. Experiments were repeated four times. In the Sixfors ${ }^{\circledR}$ reactors, DO and $\mathrm{pH}$ readings were obtained in real-time using the built-in dissolved oxygen (405 DPAS-SC-K8S/200, Mettler Toledo, Toledo, $\mathrm{OH}$ ) and pH probes (InPro 6100/220/S/N, Mettler Toledo), respectively. Samples for OD off-line measurement were obtained by syringes through a sampling port at defined time intervals. The stirring speed was $500 \mathrm{rpm}$ and the aeration rate was set to 1 volume of air per volume of medium per minute (VVM). In Sixfors three rows of six-blade Rushton flat propellers were used for stirring. Three replicate experiments were performed.

For microbioreactor experiments, $\mathrm{DO}, \mathrm{pH}$, and $\mathrm{OD}$ data were obtained on-line every $10 \mathrm{~min}$ after inoculation. Following each fermentation experiment, the volume of the culture $(150 \mu \mathrm{L})$ was harvested and the final $\mathrm{OD}_{600 \mathrm{~nm}}$ and $\mathrm{pH}$ values were measured. Calibration curves for OD readings were obtained by filling the microbioreactor with culture fluids with different biomass concentration. The $\mathrm{OD}_{600 \mathrm{~nm}}$ reading of the inoculation medium and the final $\mathrm{OD}_{600 \mathrm{~nm}}$ reading were then used to calibrate real-time OD measurement. Since the optical absorbance of PDMS changes after being dipped in water (Chang et al., 2003), the microbioreactor was filled with sterile water for more than $6 \mathrm{~h}$ before each experiment to eliminate any potential changes in optical properties. Experiments in the microbioreactor were replicated three times using independent inoculums.
S. cerevisiae ATCC 4126 batch culture experiments were cultured at $30^{\circ} \mathrm{C}$ in YPGal rich medium containing $10 \mathrm{~g} / \mathrm{L}$ yeast extract (Difco, Becton Dickinson and Company), $5 \mathrm{~g} / \mathrm{L}$ peptone (Difco), $10 \mathrm{~g} / \mathrm{L}$ glactose (Sigma-Aldrich), and $50 \mu \mathrm{g} /$ L streptomycin (Sigma-Aldrich). The standard inoculation procedure used in $S$. cerevisiae experiments was similar to the $E$. coli experimental protocol: $5 \mathrm{~mL}$ of each medium were inoculated with a single colony from an overnight streptomycin agar plate and incubated at $30^{\circ} \mathrm{C}$ on a roller drum at $60 \mathrm{rpm}$. At an $\mathrm{OD}_{600 \mathrm{~nm}}$ of about 1.0, $1.6 \mathrm{~mL}$ of culture medium was used to inoculate $30 \mathrm{~mL}$ of fresh medium in 500 $\mathrm{mL}$ baffled shake flasks and incubated at $30^{\circ} \mathrm{C}$ on the shaker until the optical density reached about 1.0. At this point the culture was diluted in fresh medium to reach an $\mathrm{OD}_{600 \mathrm{~nm}}$ of about 0.05 and used to inoculate microbioreactors. Optical on-line measurements were made every $20 \mathrm{~min}$. Growth data analysis of $S$. cerevisiae was adjusted in term of lag phases.

\section{$K_{\mathrm{L}}$ a Measurement}

The gassing-out method (Stanbury et al., 1995) was used to evaluate $K_{\mathrm{L}} a$ values in LB medium (without microbial cells) in the microbioreactor as well as in 500-mL Sixfors ${ }^{\mathbb{R}}$ bioreactors. In the Sixfors ${ }^{\circledR}$ system, nitrogen gas was continuously sparged into the medium until the oxygen concentration dropped to zero. Air was then sparged and the DO profile as a function of time was measured by the oxygen electrode. For the microbioreactor, nitrogen was flushed into the headspace over the aeration membrane until the residual DO in the medium was depleted. Then, air was pumped into the headspace and the DO recovery was recorded by the optical sensor. The $K_{\mathrm{L}} a$ values were obtain from the following expression:

$$
K_{\mathrm{L}} a=-\frac{1}{t} \ln \left[\frac{C^{*}-C_{L}}{C^{*}}\right]=-\frac{\ln (1-\mathrm{DO})}{t}
$$

where $t$ is time, $C_{\mathrm{L}}$ is the dissolved oxygen concentration in the liquid phase, $C^{*}$ is the saturated dissolved oxygen concentration, and DO $(\%)$ is the ratio of $C_{\mathrm{L}} / C^{*} \times 100$.

The DO sensor response time $T_{\mathrm{p}}$ needs to be excluded from above $K_{\mathrm{L}} a$ calculation. $T_{\mathrm{p}}$ was determined as the time needed to record $63 \%$ of a stepwise oxygen concentration change after the sensor was transferred from oxygen-free medium to a wellagitated, oxygen-saturated medium. By assuming a first-order response model, the adjusted $K_{\mathrm{L}} a$ was obtained by fitting DO data as a function of time using the following equation,

$$
1-\mathrm{DO}=\frac{T_{m} \exp \left(-t / T_{m}\right)-T_{p} \exp \left(-t / T_{p}\right)}{T_{m}-T_{p}}
$$

where $T_{\mathrm{m}}$ is the measured time constant of oxygen mass transfer, and $T_{\mathrm{m}}=1 / K_{\mathrm{L}} a$ (Zanzotto et al., 2004).

\section{Flow Simulations}

Flow patterns in the microbioreactor were simulated using CFD-ACE ${ }^{\circledR}$ software (ESI US R\&D, Inc., Columbia, MD). 
Water was used as the model fluid and the microbioreactor geometry was simplified as a cylindrical chamber of $10 \mathrm{~mm}$ diameter and $2 \mathrm{~mm}$ depth. The reactor chamber was divided into $0.7 \times 10^{6}$ structured finite elements. The three-dimensional flow in the reactor chamber was simulated by following the angular rotation of the magnetic stir bar until a steady solution was obtained. In each step of the rotation ( $1^{\circ}$ in movement, corresponding to $0.001 \mathrm{~s}$ in time step) the solution of the continuity equation and Navier-Stokes equations was obtained within 2,000 iterations; steady flow was typically established after $\sim 1,100$ rotations.

\section{RESULTS AND DISCUSSION}

\section{Mixing and Oxygenation}

The current microbioreactor design has a magnetic stirring unit for active mixing, in contrast to the previous design (Zanzotto et al., 2004) that relied on diffusion. The flow in the microbioreactor is characterized by the Reynolds number:

$$
\operatorname{Re}_{n}=\frac{D^{2} N \rho}{\mu}
$$

where $D$ is the length of the stir bar (from arm to arm), $N$ is the rotation rate for the stir bar. $\rho$ is the density of liquids, and $\mu$ is the liquid viscosity coefficient. Using water as the model medium, the Reynolds number for the microbioreactor ranges from 30 (at $180 \mathrm{rpm}$ ) to $130(850 \mathrm{rpm})$, corresponding to laminar to transitional flow regime in stirred reactors (Bin, 1984; Blanch and Clark, 1996). LB medium was measured to have a $16 \%$ higher viscosity (Ubbelohde viscometer, Technical Glass Products, Inc., Dover, NJ) than pure water and therefore has a lower Reynolds numbers when stirred at same speeds. However, the difference in viscosity did not perturb the stirring speed because of the efficient coupling between the stir bar and the magnetic drive. As an indication of the efficiency of mixing, Figure 3 illustrates the distribution of phenol red dye in the microbioreactor as a function of mixing time. The indicator color is uniformly distributed throughout the reactor indicating complete mixing within $30 \mathrm{~s}$ mixing at a stirring speed of $180 \mathrm{rpm}$. The mixing speed increases with stirring speed.

Computational fluid dynamic simulations and measurements of the oxygen mass transfer rate provide further characterization of the mixing properties. The steady state flow at $180 \mathrm{rpm}$, as simulated by CFD-ACE ${ }^{\circledR}$ (Fig. 4), reveals a secondary vertical flow caused by the horizontal stir bar rotation creating a toroidal flow pattern. This vertical flow contributes to the oxygen mass transportation from the top PDMS membrane to the DO sensor located at the bottom of the reactor. Vuppu et al. (2004) also simulated the rotation of a $\mu \mathrm{m}$-scale rotor; but their rotational flow is in the regime of very low Reynolds number (maximal $\operatorname{Re}_{\mathrm{n}}$ of $\sim 0.05$ ) and viscous forces dominate over inertial forces with poor mixing as the consequence. Flow in the present microbioreactor has a significantly higher Reynolds number $\left(\operatorname{Re}_{\mathrm{n}}\right.$ of $\left.30 \sim 130\right)$ and the convective inertial force dominates in this laminar and

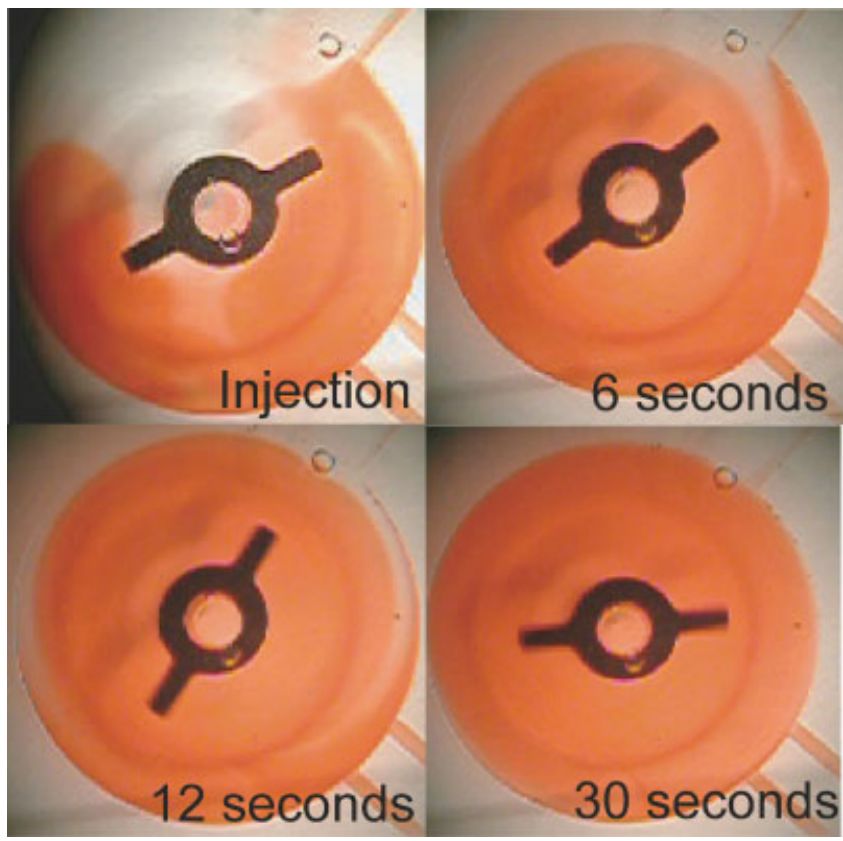

Figure 3. Photographs of mixing of phenol red dye in the microbioreactor operating with a stirring speed of $180 \mathrm{rpm}$. Note that the shadow of stir bar is projected and visible at the bottom of the chamber.

transitional flow regime. As a readily adjusted parameter, stirring speed has significant effects on mixing in this flow regime, thus allowing for manipulation of oxygenation in the microbioreactor. The general flow pattern of the secondary flow in the vertical direction superimposed on the fluid rotation retains the same features with increasing rotation speed and increases in intensity [cf. Fig. 4b and e vs. Fig. 4d and $\mathrm{f}$ ].

The oxygen mass transfer rate in bioreactors is a critical parameter of microbial fermentation. The volumetric masstransfer coefficient, $K_{\mathrm{L}} a$, is often used as a measure of the aeration capacity of a bioreactor. Figure $5 \mathrm{a}$ and $\mathrm{b}$ show the recovery curves of dissolved oxygen as well as the DO sensor response curve as measured by the gassing out method. First order response model for the gassing method, Equation 2, is proven by the linearity (in logarithm scale) of curves shown in Figure 5. The DO electrode used in the Sixfors ${ }^{\circledR}$ has a response time $T_{\mathrm{p}}$ of $12.7 \mathrm{~s}$ at a stirring speed of $500 \mathrm{rpm}$ and a sparging rate of 1 VVM. The $K_{\mathrm{L}} a$ in Sixfors ${ }^{\circledR}$ bioreactor is calculated as $48.2 / \mathrm{h}$. The response time of the DO optical sensor in the microbioreactor is $33.6 \mathrm{~s}$ (Fig. 5b). At a $180 \mathrm{rpm}$ stirring speed, the $K_{\mathrm{L}} a$ value in the microbioreactor is $22.2 / \mathrm{h}$, which is lower than the $K_{\mathrm{L}} a$ value of $48.2 / \mathrm{h}$ in the Sixfors ${ }^{\circledR}$ reactor $(500 \mathrm{rpm}, 1 \mathrm{VVM})$. Higher oxygen transfer rates can be obtained in the microbioreactor with higher stirring speeds (Fig. 6); for example, at a stirring speed of $700 \mathrm{rpm}$, the $K_{\mathrm{L}} a$ is $61.9 / \mathrm{h}$, exceeding the value obtained in the bench top reactors. Standard deviations in the measurements are less than $2 \%$.

In the microbioreactor, oxygen is supplied to the medium by oxygen diffusion through the PDMS membrane to the liquid phase. There is no gas sparging through the liquid 

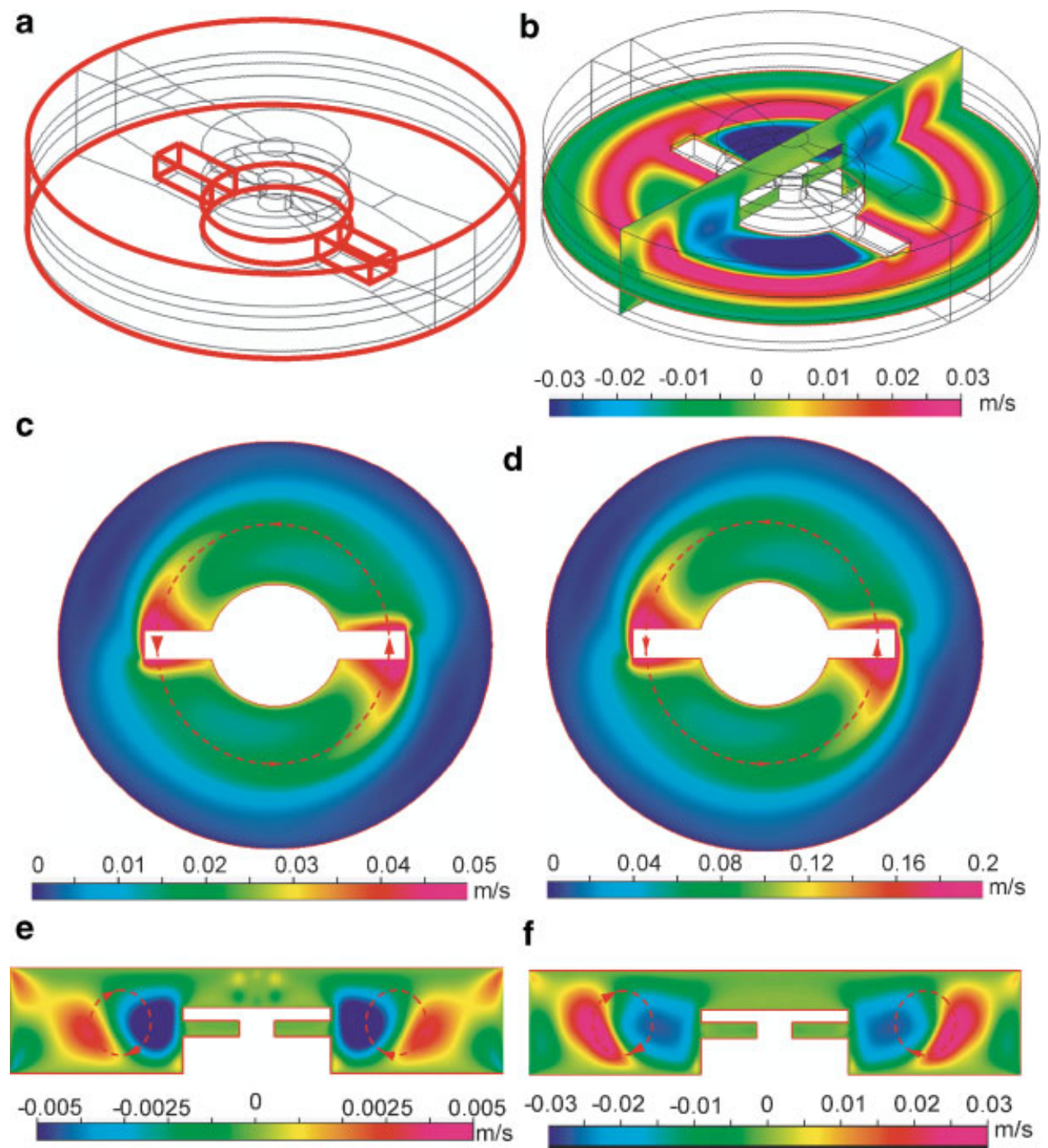

Figure 4. CFD-ACE ${ }^{\circledR}$ simulation of medium flow in the microbioreactor operating at stirring speeds of $180 \mathrm{rpm}$ and $700 \mathrm{rpm}$. a: Simplified sketch of the reactor chamber and the stir bar. b: Three-dimensional view of the vertical flow velocity distribution at a horizontal plane in the center and a vertical plane $0.6 \mathrm{~mm}$ above the bottom of the microbioreactor. Stirring speed is $700 \mathrm{rpm}$. $\mathbf{c}$ and $\mathbf{d}$ : Comparison of the magnitude of flow velocity at a vertical plane $0.6 \mathrm{~mm}$ above the bottom of the microbioreactor at stirring speed of (c) $180 \mathrm{rpm}$ and (d) $700 \mathrm{rpm}$. e and f: Comparison of the vertical flow circulation at a horizontal plane in the microbioreactor at stirring speed of (e) $180 \mathrm{rpm}$ and (f) $700 \mathrm{rpm}$. The general flow pattern of the secondary flow in the vertical direction superimposed on the fluid rotation retains the same features with increasing rotation speed and increases in intensity as reflected by the difference in scales. The red dashed curves in the figures show the direction of stirrer movement and the vertical circulation of flow at the given cross-section.

medium and no moving gas-liquid interface, as a consequence, the specific mass-transfer area, $a$, stays constant. Similar to surface-aerated bioreactors (without bubble entrainment), the improvement in $K_{\mathrm{L}} a$ values in the microbioreactor at higher stirring speeds results from better mixing and distribution of dissolved oxygen in the liquid phase. The overall oxygen mass-transfer resistance $1 / K_{\mathrm{L}, \text { overall }}$ in the microbioreactor is the sum of three resistances in series:

$$
\frac{1}{K_{\mathrm{L}, \text { overall }}}=\frac{1}{K_{\mathrm{L}}}+\frac{\delta}{D_{\mathrm{PDMS}} H}+\frac{1}{K_{\mathrm{G}}}
$$

where $1 / \mathrm{K}_{\mathrm{L}}$ is the mass-transfer resistance in liquid phase, $\delta /$ $\left(D_{\text {PDMS }} H\right)$ is the diffusion resistance through PDMS, and 1/ $K_{\mathrm{G}}$ represents the diffusion resistance in air. The mass transfer coefficients and the simple film model are typically used in cases of turbulent flow, such as in traditional large scale fermentors. Nevertheless, with appropriate definition of the mass transfer coefficients (Bird et al., 2002), the concept also applies for the flow conditions in the microbioreactor and it has been applied to similar issues in microtiter plates (Hermann et al., 2002). In particular, it provides a useful means for evaluating the relative importance of the different resistances to oxygen transport into the microbioreactor (Eq. 4). The diffusivity of oxygen in air is significantly higher than in PDMS and water, thus $1 / K_{\mathrm{G}}$ can be neglected compared with the other terms in Equation 4. The PDMS membrane has a thickness $\delta$ of $100 \mu \mathrm{m}$, and the diffusivity of oxygen in PDMS $D_{\text {PDMS }}$ is $3.4 \times 10^{-5} \mathrm{~cm}^{2} / \mathrm{s}$ (Merkel et al., 2000). The solubility of oxygen in PDMS and water are $1.69 \mathrm{mmol} / \mathrm{L}$ (Merkel et al., 2000) and $0.228 \mathrm{mmol} / \mathrm{L}$ (Perry and Green, 1984), respectively. The Henry's constant $H$, the solubility ratio of oxygen in PDMS 


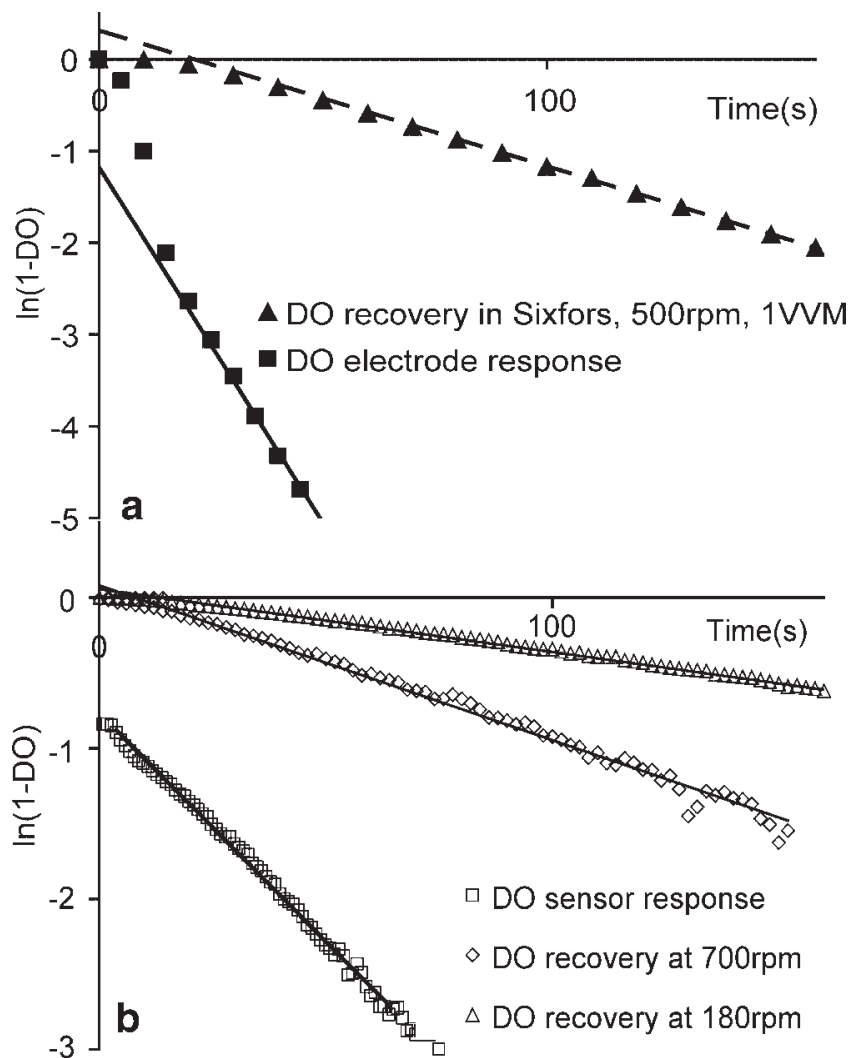

Figure 5. Recovery of dissolved oxygen curves as well as the DO sensor response curves in (a) SixFors ${ }^{\circledR}$ reactor (stirring speed of $500 \mathrm{rpm}$ and sparging rate of $1 \mathrm{VVM}$ ) and (b) microbioreactor (stirring speed of 180 and $700 \mathrm{rpm})$.

and water, equals to 7.4. With a fixed surface aeration area of $90 \mathrm{~mm}^{2}$, the overall mass transfer coefficient, $K_{\mathrm{L} \text {,overall }}$, ranges from $0.037 \mathrm{~m} / \mathrm{h}(180 \mathrm{rpm})$ to $0.13 \mathrm{~m} / \mathrm{h}(850 \mathrm{rpm})$. The net mass transfer coefficient in water, $K_{\mathrm{L}}$, ranges from $0.039 \mathrm{~m} / \mathrm{h}(180 \mathrm{rpm})$ to $0.15 \mathrm{~m} / \mathrm{h}(850 \mathrm{rpm})$. These values are consistent with or slightly lower than reported literature values for surface-aerated stirred-tank bioreactors (Kawase and Moo-Young, 1990; Kamen et al., 1995). The variation in

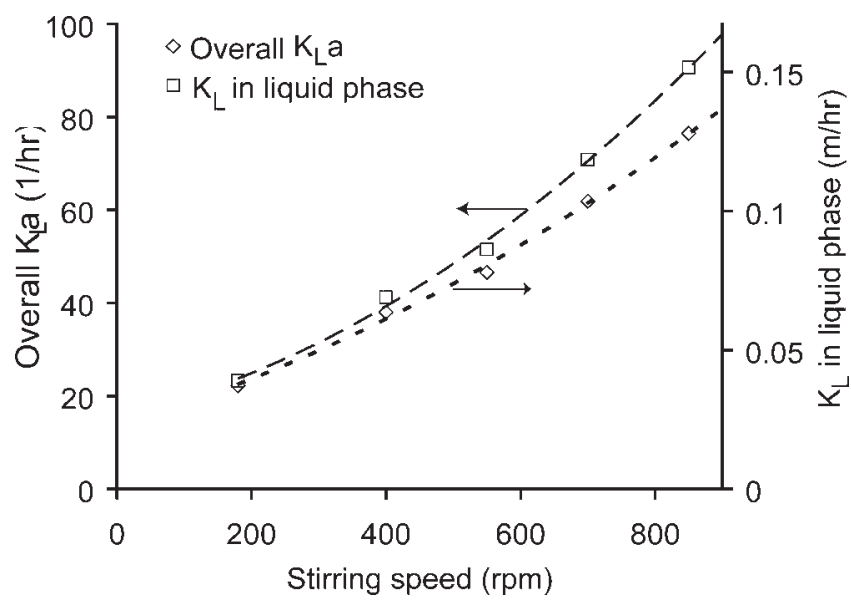

Figure 6. Oxygen volumetric mass transfer coefficients as a function of stirring speed in the microbioreactor.
$K_{\mathrm{L}}$ with changing stirring speed (Fig. 6) is similar to the change observed with varying shaking frequency in microtiter plates (Hermann et al., 2002), which have wells of similar volume $(200 \mu \mathrm{L})$ as the present microbioreactor.

\section{Batch Fermentation in Microbioreactors}

We investigated $E$. coli growth kinetics in the microbioreactor to demonstrate the feasibility of microbial cultivation in the microbioreactor. Triplicate fermentation experiments were carried out in the microbioreactor at both 180 and $700 \mathrm{rpm}$ stirring speed. The observed OD, pH, and DO values within the microbioreactor (Fig. 7) demonstrate the reproducibility of cell growth in the microbioreactor under different growth conditions.

As a result of the inoculation preparation protocol, the $E$. coli cells start to grow immediately without any observable lag phase. Consistent with the high growth rate of cells during exponential growth phase, the high demand for oxygen is reflected by the rapid decrease in DO level in the culture medium. Although avoiding oxygen depletion is the best interest for many specific bioprocesses, we intentionally chose oxygen-limited growth conditions for the cultivation of microbial cells to obtain more information about the microbioreactor itself and to better benchmark the microbioreactor. High-capability oxygenation is not the major
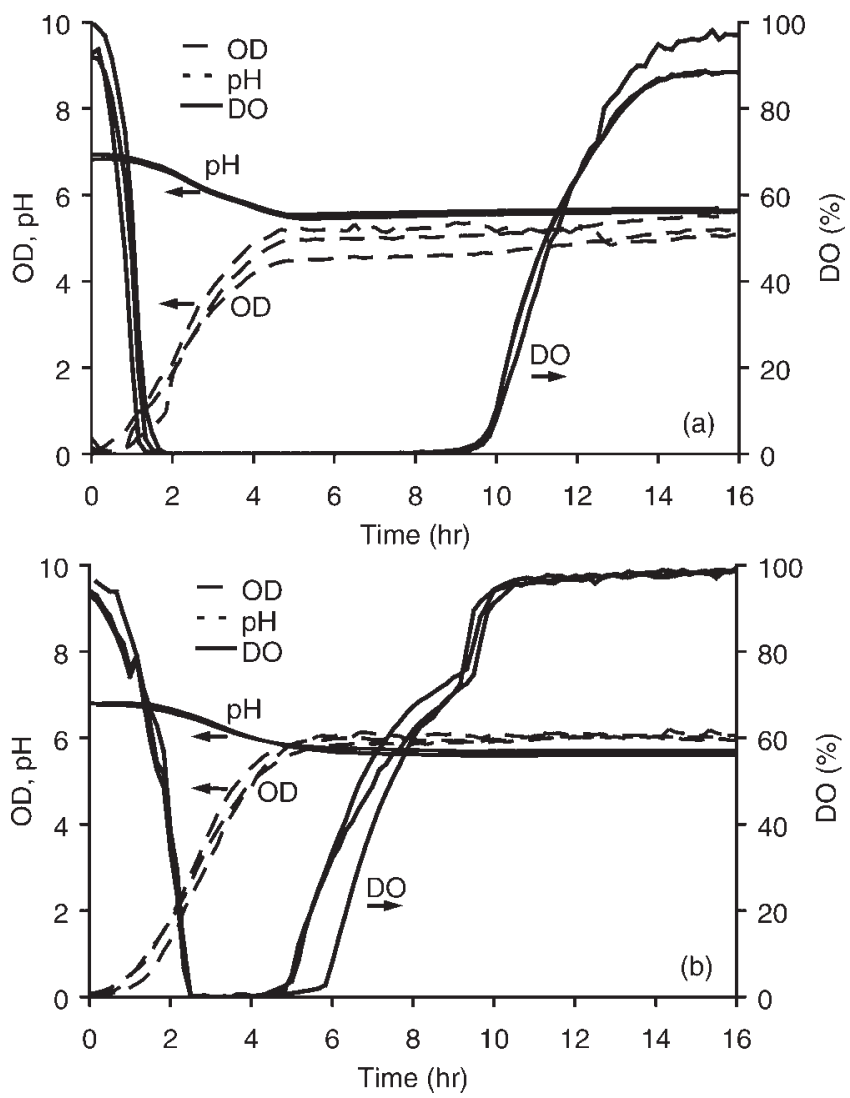

Figure 7. Replicate fermentations $(n=3)$ of E. coli FB21591 in LB medium with stirring speed of (a) $180 \mathrm{rpm}$ and (b) $700 \mathrm{rpm}$. 
pursue for our microbioreactor; the reproducibility and the correlation of microbioreactor to conventional benchscale bioreactors are more important for a potentially applicable high-throughput platform with microliter volume and on-line measurements.

With a stirring speed of $180 \mathrm{rpm}$, the DO in the microbioreactor completely depletes at around $1.5 \mathrm{~h}$ of the fermentation and does not recover until after $9 \mathrm{~h}$ (Fig. 7a), when the cells enter late-stationary phase and become limited in carbon source. During this period, as a consequence of anaerobic fermentation the $\mathrm{pH}$ level in the medium decreases significantly until cells reach stationary phase at around $6 \mathrm{~h}$.

Consistent with the $K_{\mathrm{L}} a$ measurements, improved oxygenation is observed at higher stirring speeds (Fig. 7b). With a stirring speed of $700 \mathrm{rpm}$, the DO level in the microbioreactor depletes after $2.5 \mathrm{~h}$ of the fermentation and recovers at $\sim 5.5 \mathrm{~h}$, much earlier than the recovery time in fermentations with stirring speed of $180 \mathrm{rpm}$. With better oxygenation cells grow faster and reach higher biomass concentrations. The final OD level at $700 \mathrm{rpm}$ stirring reaches $6.2 \pm 0.17$ in contrast to the final OD value of $5.3 \pm 0.2$ at $180 \mathrm{rpm}$.

The reproducibility and flexibility of the microbioreactor is also demonstrated by triplicate $S$. cerevisiae batch culture experiments (Fig. 8). S. cerevisiae ATCC 4126 cells were cultured in YPGal medium with $10 \mathrm{~g} / \mathrm{L}$ glactose. The stirring speed is $700 \mathrm{rpm}$ in these experiments, and similar standard inoculation procedure is applied. Dissolve oxygen depletes at around $10 \mathrm{~h}$ into the culture, and recovers around $15 \mathrm{~h}$, when cells have used the nutrients and enter the stationary phase. Yeast cells reached a maximum $\mathrm{OD}_{600}$ of $6.87( \pm 0.07)$. At this population density, the $\mathrm{pH}$ and the dissolved oxygen concentration are $6.82( \pm 0.09)$ and $11.2( \pm 1.3) \%$, respectively.

\section{Benchmarking With Conventional Lab-Scale Bioreactors}

We compared E. coli fermentation results in the microbioreactor with data from conventional bioreactors, including Sixfors ${ }^{\circledR}$, test tubes, and shake flasks (Fig. 9) for benchmarking purpose. Both the time profile and the standard

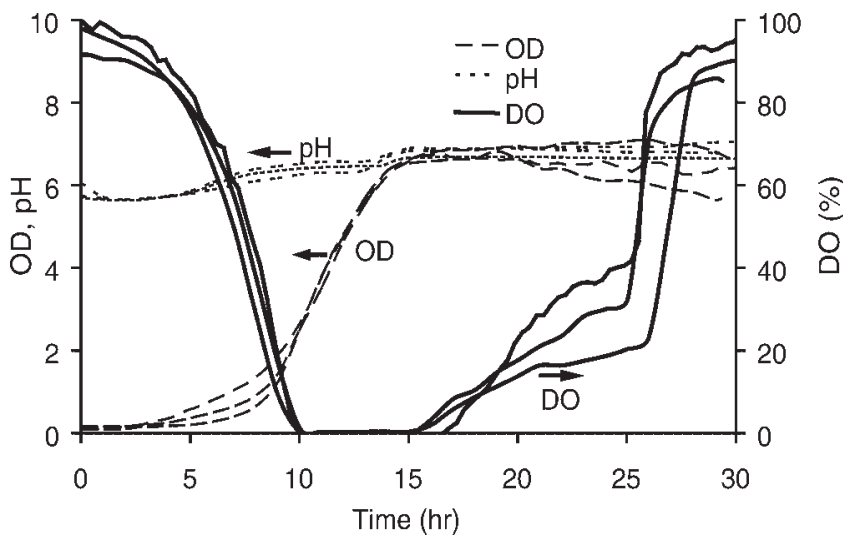

Figure 8. Growth data: replicate fermentations $(n=3)$ of $S$. cerevisiae in YPGal medium.

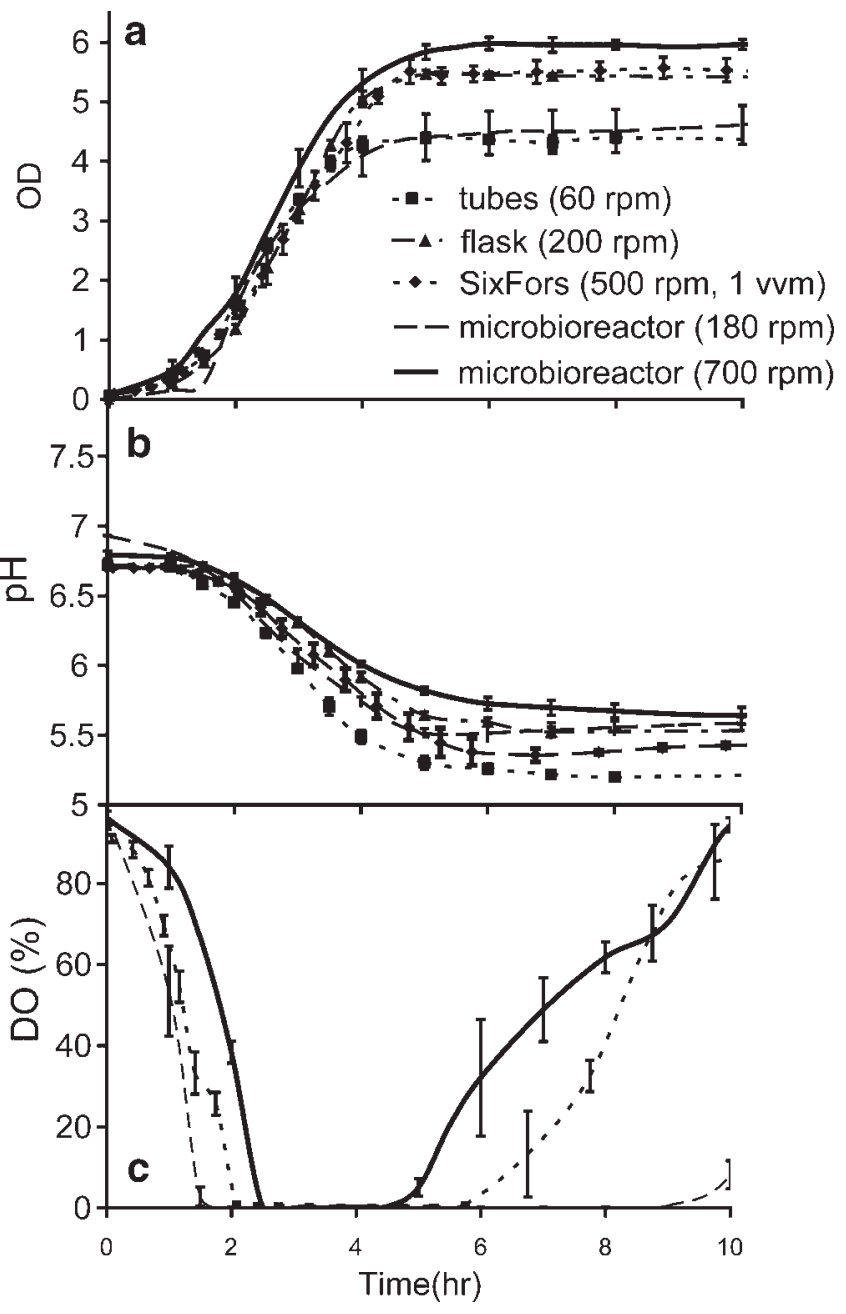

Figure 9. Comparison of E. coli FB21591 growth kinetics in LB medium in the microbioreactor $(n=3$, stirring speed at 180 and $700 \mathrm{rpm}$, respectively) with conventional bioreactors including test tubes $(n=4)$, shake flasks $(n=4)$, and Sixfors $(n=3)$. Each growth curve represents the average value with standard deviation from $\mathrm{n}$ replicate experiments carried out in separate bioreactors. (a) OD; (b) pH; (c) DO.

deviation of the OD curves obtained in the microbioreactor are comparable with other conventional bioreactors (Fig. 9a). The highest biomass was obtained with the microbioreactors stirred at $700 \mathrm{rpm}$, followed by the Sixfors ${ }^{\circledR}$ bioreactors (500 rpm, 1 VVM) and the shake flasks (200 rpm shaking speed), and then by the microbioreactor stirring at $180 \mathrm{rpm}$. These results are consistent with the oxygenation characteristics in the reactors, including both the $K_{\mathrm{L}} a$ data and the DO profiles during batch fermentation (Fig. 9c). As a dynamic process, the recovery of DO in bioreactors happens when glucose becomes limiting, and this recovery is significantly slower in less oxygenated reactors (microbioreactor operating at $180 \mathrm{rpm}$ ), and cells grow anaerobically for longer period of time.

The $\mathrm{pH}$ curves for the microbioreactor and those from all other bioreactors (Fig. 9b), as well as the DO profiles (Fig. 9c) from the microbioreactor and the Sixfors ${ }^{\circledR}$ are all similar. In our experiments, test tubes and shake flasks are not equipped 


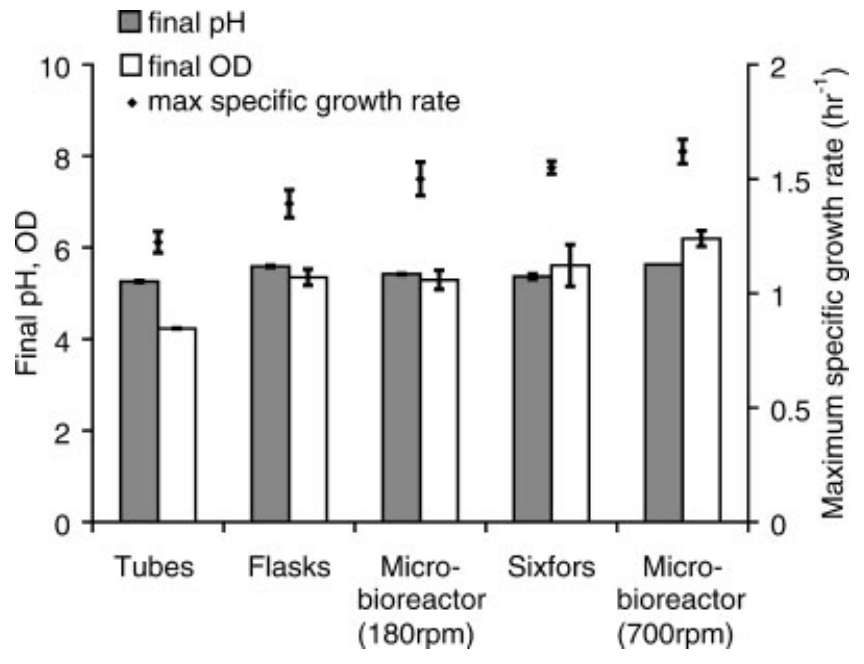

Figure 10. Comparison of final $\mathrm{OD}, \mathrm{pH}$, and maximal growth rate in $E$. coli FB21591 batch cultures in different bioreactors.

with DO sensors; but the oxygenation can be estimated by the growth rate and final OD level (Fig. 10). Cell growth in test tubes has the lowest final OD $(4.23 \pm 0.01)$, which can be attributed to the low oxygenation level. Gupta and Rao (2003) reported a $K_{\mathrm{L}} a$ value in baffled shake flasks with $100 \mathrm{~mL}$ filling volume and shaking speed of $250 \mathrm{rpm}$ as $\sim 59.2 / \mathrm{h}$. Because a lower shaking speed and a larger filling volume are used here, the $K_{\mathrm{L}} a$ value of our shake flasks would be expected to be less than the reported value. This assumption is consistent to our experimental data: cell growth rate, final biomass concentration, and final $\mathrm{pH}$ level in shake flasks $(1.39 \pm 0.06 / \mathrm{h}, 5.35 \pm 0.18$, and $5.59 \pm 0.02$, respectively) are close to the values obtained in Sixfors ${ }^{\circledR}(1.55 \pm 0.03 / \mathrm{h}$, $5.37 \pm 0.06$, and $5.61 \pm 0.47$, respectively). Similarly, the reproducibility of the optical density data obtained in the microbioreactor $(5.29 \pm 0.2$ at $180 \mathrm{rpm}$ and $6.20 \pm 0.17$ at $700 \mathrm{rpm}$ ), is comparable to that from flasks and bench-scale bioreactors. The ability to change mixing in the microbioreactor by different stirring speed enables control of oxygenation and also allows varying growth kinetics ranging from shake flasks conditions to those characterized conventional bench-scale stirred-tank bioreactors, such as Sixfors ${ }^{\circledR}$.

\section{CONCLUSIONS}

We have designed and fabricated a $150 \mu \mathrm{L}$ membraneaerated and actively mixed microbioreactor in PMMA and PDMS. Optical sensors for OD, $\mathrm{pH}$, and DO real time measurements were integrated into the microbioreactor. PMMA and PDMS were chosen as materials for the bioreactor, for their good optical transparency to visible light, biocompatibility and mechanical rigidity, low cost, as well as their potential for large-scale manufacturing; the latter characteristics should allow the microbioreactor to be disposable.

Active mixing facilitates oxygenation in membraneaerated bioreactors and enables larger culture volume for bio-analysis, while still maintaining reasonable levels of oxygenation for cell growth. The volumetric mass-transfer coefficient, $K_{\mathrm{L}} a$, was characterized to range from 20 to $75 / \mathrm{h}$, which are comparable to those from conventional benchscale bioreactors. Growth kinetics, in terms of time profiles and final values of $\mathrm{OD}, \mathrm{pH}$, and $\mathrm{DO}$ measurements, were reproducible for the micro batch bioreactors and corresponded closely to those observed with conventional experimental methods, including test tubes, shake flasks, and bench-scale stirred tank bioreactors (Sixfors ${ }^{\mathbb{R}}$ ). This ability to mirror observations in conventional systems coupled with reproducible cell growth, small reactor volumes, and on-line measurements of $\mathrm{DO}, \mathrm{pH}$, and $\mathrm{OD}$ suggest that microbioreactors could be useful tools for high throughput bioprocessing.

The authors gratefully acknowledge the DuPont-MIT Alliance (DMA) for funding. Dr. Nicolas Szita is also thankful to the Swiss National Science Foundation for additional funding.

\section{References}

Bin AK. 1984. Mass transfer to the free interface in stirred vessels. Chem Eng Commun 31:155-183.

Bird RB, Steward WE, Lightfoot EN. 2002. Transport phenomena. New York: John Wiley \& Sons, Inc.

Blanch HW, Clark DS. 1996. Biochemical engineering. New York: Marcel Dekker, Inc.

Boccazzi P, Zanzotto A, Szita N, Bhattacharya S, Jensen KF, Sinskey AJ. 2005. Gene expression analysis of Escherichia coli grown in miniaturized bioreactor platforms. Appl Microbio Biotechol 68:518 532.

Chang W-J, Akin D, Sedlak M, Ladisch MR, Bashir R. 2003. Poly(dimethylsiloxane) (PDMS) and silicon hybrid biochip for bacterial culture. Biomed Microdev 5(4):281-290.

Duetz WA, Witholt B. 2001. Effectiveness of orbital shaking for the aeration of suspended bacterial cultures in square-deepwell microtiter plates. Biochem Eng J 7:113-115.

Duetz WA, Rüedi 1, Hermann R, O’Connor K, Büchs J, Witholt B. 2000. Methods for intense aeration, growth, storage, and replication of bacterial strains in microtiter plates. Appl Env Microbiol 66:2641-2646.

Gupta A, Rao G. 2003. A study of oxygen transfer in shake flasks using a noninvasive oxygen sensor. Biotechol Bioeng 84(3):351-358

Harms P, Kostov Y, Rao G. 2002. Bioprocess monitoring. Curr Opin Biotechol 13:124-127.

Hermann R, Lehmann M, Büchs J. 2002. Characterization of gas-liquid mass transfer phenomena in microtiter plates. Biotechol Bioeng 81(2):178-186.

John GT, Klimant I, Wittmann C, Heinzle E. 2003a. Integrated optical sensing of dissolved oxygen in microtiter plates: A novel tool for microbial cultivation. Biotechol Bioeng 81(7):829-836.

John GT, Goelling D, Klimant I, Schneider H, Heinzle E. 2003b. pH-sensing 96-well microtiter plates for the characterization of acid production by dairy starter cultures. J Dairy Res 70(3):327-333.

Kamen A, Garnier A, Andre G, Archambault J, Chavarie C. 1995. Determination of mass transfer parameters in surface aerated bioreactors with bubble entrainment. Chem Eng J 59:187-193.

Kawase Y, Moo-Young M. 1990. Mass transfer at a free surface in stirred tank bioreactors. Trans IChemE 68:189-194.

Kermis HR, Kostov Y, Rao G. 2003. Rapid method for the preparation of a robust optical pH sensor. Analyst 128:1181-1186. 
Kim JW, Lee YH. 1998. Development of microfermenter Chip. J Korean Phys Soc 33:S462-S466.

Kostov Y, Harms P, Randers-Eichhorn L, Rao G. 2001. Low-cost microbioreactor for high-throughput bioprocessing. Biotechol Bioeng 72(3):346-352.

Kumar S, Wittmann C, Heinzle E. 2004. Minibioreactors. Biotechol Lett 26:1-10.

Lamping SR, Zhang H, Allen B, Shamlou PA. 2003. Design of a prototype miniature bioreactor for high throughput automated bioprocessing. Chem Eng Sci 58:747-758.

Maharbiz MM, Holtz WJ, Sharifzadeh S, Keasling JD, Howe RT. 2003. A microfabricated electrochemical oxygen generator for high-density cell culture arrays. J MEMS 12(5):590-599.

Maharbiz MM, Holtz WJ, Howe RT, Keasling JD. 2004. Microbioreactor arrays with parametric control for high-throughput experimentation. Biotechol Bioeng 85(4):376-381.

Merkel T, Bondar V, Nagai K, Freeman B, Pinnau I. 2000. Gas sorption, diffusion, and permeation in poly(dimethysiloxane). J Polym Sci Pt B Polym Phys 38(3):415-434.

Perry R, Green D. 1984. Perry's chemical engineering handbook. Chicago: R.R. Donnelley \& Sons Company.

Puskeiler R, Kaufmann K, Weuster-Botz D. 2005. Development, parallelization, and automation of a gas-inducing milliliter-scale bioreactor for high-throughput bioprocess design (HTBD). Biotechol Bioeng 89(5): 512-523.

Rao G. 2002. Feb 28, 2002. Bioreactor and bioprocessing technique. USA patent US 2002/0025547 A1.

Stanbury PF, Whitaker A, Hall SJ. 1995. Principles of fermentation technology: Oxford, UK: Elsevier Science Ltd. pp 243-253.
Tolosa L, Kostov Y, Harms P, Rao G. 2002. Noninvasive measurement of dissolved oxygen in shake flasks. Biotechol Bioeng 80(5):594-597.

Van der Weide DW, Blattner FR. 2002. Microfabricated microbial growth assay method and apparatus. US patent US 2002/0197709 A1.

Vuppu AK, Garcia AA, Saha SK, Phelan PE, Hayes MA, Calhoun R. 2004. Modeling microflow and stirring around a microrotor in creeping flow using a quasi-steady-state analysis. Lab Chip 4:201-208.

Walther I, van der Schoot BH, Jeanneret S, Arquint P, de Rooij NF, Gass V, Bechler B, Lorenzi G, Cogoli A. 1994. Development of a miniature bioreactor for continuous culture in a space laboratory. J Biotechol 30(1):21-32.

Walther I, van der Schoot BH, Boillat M, Cogoli A. 1999. Microtechnology in space bioreactors. Chimia 53:75-80.

Walther I, van der Schoot BH, Boillat M, Cogoli A. 2000. Performance of a miniaturized bioreactor in space flight: Microtechnology at the service of space Biology. Enz Microbial Techol 27:778-783.

Weiss S, John GT, Klimant I, Heinzle E. 2002. Modeling of mixing in 96-well microplates observed with fluorescence indicators. Biotechol Prog 18:821-830.

Weuster-Botz D, Altenbach-Rehm J, Aenold M. 2001. Parallel substrate feeding and pH-control in shaking-flasks. Biochem Eng J 7:163-170.

Weuster-Botz D, Stevens S, Hawrylenko A. 2002. Parallel-operated stirredcolumns for microbial process development. Biochem Eng J 11:69-72.

Wittmann C, Kim HM, John G, Heinzle E. 2003. Characterization and application of an optical sensor for quantification of dissolved $\mathrm{O}_{2}$ in shake-flasks. Biotechol Lett 25(5):377-380.

Zanzotto A, Szita N, Boccazzi P, Lessard P, Sinskey AJ, Jensen KF. 2004. A membrane-aerated microbioreactor for high-throughput bioprocessing. Biotechol Bioeng 85(4):376-381. 